\title{
Escuelas Normales y formación de educadores desde una Educación para niños con discapacidad ${ }^{1}$
}

\author{
Normal Schools and teacher training from Education for children \\ with disabilities
}

\section{Escolas Normais e formação de professores para uma Educação de crianças com deficiência}

\author{
Mônica María Farid Rahme ${ }^{2}$ \\ Universidad Federal de Ouro Preto (Brasil) \\ Grupo Investigación HISULA
}

Recepción: 02/02/2015

Evaluación: 27/05/2015

Aceptación: 02/06/2015

Artículo de investigación científica y tecnológica

\section{RESUMEN}

Este artículo discute temas relacionados a investigación bibliográfica, elementos la formación de profesores en el ámbito de históricos relacionados a la formación de la educación especializada brasilera. Para profesores en Brasil y su articulación con ello, retoma datos del período colonial de la educación de alumnos que presentan los primeros años de la República hasta los días actuales, cuando la formación particularidades físicas, motoras, sensoriales, psíquicas y / o cognitivas. Para de profesores concede el desafío de la elaboración de este trabajo, se defiende hacer concreta una educación que se espera para todos. Con esta finalidad, el artículo explora, por medio de una la hipótesis de que la constitución de instituciones educativas destinadas a este público y la emergencia posterior de las

1 Este artículo sistematiza temas presentados en la Conferencia realizada por la autora en la Cátedra de Historia y Prospectiva de la Universidad Latinoamericana, el día 09 de abril en la Universidad Pedagógica y Tecnológica de Colombia (UPTC). En la presentación inicial se añadieron nuevos datos así como el levantamiento de la investigación sobre diversidad e inclusión en los currículos de licenciatura de la Universidad Federal de Ouro Preto (UFOP), realizada por la autora junto a la Profesora Doctora Margareth Diniz y al Profesor Doctor Ubiratan Garcia Vieira, y a los licenciados Iasmin Cardoso Raimundi Eliana do Nascimento Libanio Maia y Ricardo Brandão. Por otra parte, este artículo es el resultado del proyecto de investigación "la Formación de las maestras rurales en Colombia y Brasil. Perspectivas de estudios comparados en Educación SGI 1730", del grupo de investigación HISULA de la Universidad Pedagógica y Tecnológica de Colombia, financiado por la Dirección de investigaciones.

2 Doctora en Educación por la Facultad de Educación - Universidad de São Paulo. Es profesora e investigadora de la Universidad Federal de Ouro Preto. Dirección para correspondencia: Rua do Seminário, S/N - Centro - Mariana, Minas Gerais, Brasil. CEP: 35420-000. E-mail: monica@ichs.ufop.br 
perspectivas de integración e inclusión escolar, condujeron al delineamiento de demandas específicas para los procesos de formación inicial de profesores, que se modifican en la medida en que suceden cambios en la orientación de la política educacional. Por último, se presentan brevemente, datos de una investigación empírica sobre la presencia de la temática de la deficiencia en los currículos de los cursos de licenciatura de una universidad pública, situada en el estado de Minas Gerais. A partir de los puntos tratados, se buscan destacar los desafíos presentes en la formación de profesores en un contexto inclusivo y la necesidad de estructurar procesos de formación que articulen efectivamente teoría y práctica.

Palabras clave: Revista Historia de la Educación latinoamericana, Formación de profesores, educación especializada, deficiencia, inclusión escolar.

\footnotetext{
ABSTRACT

This article discusses issues related to the training of teachers in the field of Brazilian special education. For this purpose, it takes data from the colonial period, from the early years of the Republic until today, when teacher training grants the challenge of specifying an education expected for everyone. To this end, the article explores, through bibliographic research, historical items related to teacher training in Brazil and its articulation with the education of students who have physical, motor, sensory, mental and/or cognitive characteristics. For the preparation of this paper, it is defended the hypothesis consisting of the establishment of educational institutions aimed to this
}

public and the subsequent emergence of integration prospects and school inclusion led to the delineation of specific demands for the processes of initial teacher training, which are modified to the extent that changes occur in the orientation of educational policy. Finally, it briefly presents empirical research data on the theme of deficiency in the curricula of undergraduate courses at a public university located in the state of Minas Gerais. From the points discussed, it is intended to highlight the challenges in the training of teachers in an inclusive context and the need to structure formation processes that effectively articulate theory and practice.

Keywords: Journal History of Latin American education, teacher training, special education, disability, school inclusion.

\section{RESUMO}

Este artigo discute questões relacionadas à formação de professores no âmbito da educação especializada brasileira. Para tanto, retoma-se dados do período colonial, dos primeiros anos da República até alcançar os dias atuais, quando a formação de professores se depara com o desafio de tornar concreta uma educação que se pretende para todos. Com esta finalidade, o artigo explora, por meio de uma pesquisa bibliográfica, elementos históricos relacionados à formação de professores no Brasil e sua articulação com a educação de alunos que apresentam particularidades físicas, motoras, sensoriais, psíquicas e/ou cognitivas. Para a elaboração desse percurso, defende-se a hipótese de que a constituição de instituições educativas 
destinadas a esse público e a emergência posterior das perspectivas de integração e inclusão escolar conduziram ao delineamento de demandas específicas para os processos de formação inicial de professores, que se modificam à medida em que ocorrem mudanças na orientação da política educacional. Por fim, apresenta-se, brevemente, dados de uma investigação empírica sobre a presença da temática da deficiência nos currículos dos cursos de licenciatura de uma universidade pública, situada no estado de Minas Gerais. A partir dos pontos abordados, procura-se destacar os desafios presentes na formação de professores em um contexto inclusivo e a necessidade de estructurar procesos de formación que articulen efectivamente teoría y práctica.

Palavras-chave: Revista Historia de la Educación latinoamericana, Formação de professores, educação especializada, deficiência, inclusão escolar.

\section{INTRODUCCIÓN}

La educación escolar de personas con deficiencia en Brasil, presenta un percurso similar a lo que sucedió en los países europeos que fueron precursores en esta área, iniciándose por la escolarización de los estudiantes ciegos y sordos para, posteriormente, instituir espacios educativos para otros grupos que presentaban particularidades cognitivas, psíquicas, físicas y motoras. ${ }^{3}$

Repasando esta trayectoria, se verifica que la oferta de escolarización para estos grupos no se encontraba directamente articulada a la institucionalización de espacios de formación profesional para educadores, siendo necesarios algunos años para que una proposición en esta perspectiva se delinease vía disciplinas específicas, Escuelas Normales, instituciones de formación, cursos de licenciatura, entre otros.

El objetivo de este artículo, es relacionar las concepciones educacionales presentes en la historia de la educación especializada brasilera y la constitución de procesos más amplios de formación docente en el país. Su justificación se relaciona al hecho de la temática de la educación de alumnos con deficiencia haberse concentrado durante muchas décadas en espacios limitados de formación de profesores - las asignaturas situadas en el área de Educación Especial - no atienden a la mayor parte de los estudiantes que tenían como perspectiva actuar en la docencia. Esa realidad pasa a ser alterada a principio de las dos últimas décadas.

Por tanto, realizamos una investigación bibliográfica, enfocando el trabajo de autores brasileros del campo de Educación Especial, lo que nos permitió ubicar las primeras iniciativas que fueron desarrolladas en esta perspectiva desde el período Brasil-Colonia y después de la proclamación de la República. Luego,

3 Marcos José da Silveira Mazzota. Educação especial no Brasil: História e políticas públicas. (São Paulo: Cortez, 2005$),$ p. 27. 
analizamos las décadas siguientes, destacando asuntos que eran tratados en los procesos de formación de educadores en un contexto de integración escolar y, por último, situamos los desafíos que se colocan actualmente para las asignaturas de licenciatura, cuando se defiende una educación escolar que sea única para todos.

En la última parte del artículo, consideramos datos de una investigación documental y empírica sobre la temática de la deficiencia en los currículos de diez asignaturas de licenciatura, en el contexto de una universidad pública brasilera, distribuidos en dos campos. Se tratan de las siguientes asignaturas: Artes Escénicas, Ciencias Biológicas, Filosofía, Física, Historia, Letras, Matemática, Música, Pedagogía, Química. A partir de esta investigación, verificamos que siete asignaturas obligatorias, trataban directa o indirectamente temáticas relacionadas a la deficiencia en uno de los campos; y en el otro, apenas una asignatura que tenía alguna relación con la temática de la deficiencia. Con base en estos datos, se puede indicar que el tema de la deficiencia no se encuentra totalmente ausente de las asignaturas de licenciatura investigadas, aunque su presencia sea aún incipiente y puntual.

Pasaremos, entonces, a discutir la institucionalización de la educación especializada en Brasil-Colonia y el período posterior a la proclamación de la República, destacando sus principales conexiones con el problema propuesto.

\section{La institucionalización de la educación especializada en Brasil-Colonia: una oferta dirigida a alumnos ciegos y sordos}

En Brasil, la primera acción que registra una política destinada a la educación de personas con discapacidad fue dirigida a los no videntes. Se trata de un proyecto de creación de la "Silla de profesores de Primeras Letras para la Enseñanza de Ciegos y Sordo Mudos, en las Escuelas de la Corte y de las Capitales de las Provincias", presentado en 1835, por el Consejero Cornélio Ferreira França, diputado de la Provincia de Bahía. Pero, el mandato del Consejero terminó antes de que el proyecto fuera aprobado. Esta sería, así, una acción gubernamental para la formación de profesores. ${ }^{4}$ Pero, como dice Januzzi ${ }^{5}$, fueron necesarios cerca de 19 años más para que se iniciara una atención en esta perspectiva.

Los dos primeros institutos para educación de niños con discapacidad en Brasil son del período del Imperio. Son muy similares a los institutos franceses que se constuyeron después de su Revolución. Así, el Imperial Instituto de Meninos Cegos fue construído por el Emperador Don Pedro II, en 1854, en la ciudad de Río de Janeiro. Este instituto era muy parecido al Institut National des Jeunes Sourds, fundado en París, en 1784 (70 años antes). El Imperial Instituto de

4 Francisco Mendes Lemos y Paulo Felicissimo Ferreira, Instituto Benjamín Constant: uma história centenária (Río de Janeiro: Instituto Benjamín Constant, 1995) 200.156.28.7/Nucleus/media/common/Downloads_Historia_IBC.doc (10 de marzo, 2014).

5 Gilberta de Martino Jannuzzi. A educação do deficiente no Brasil: Dos primórdios ao início do século XXI. (Campinas (SP): Autores Associados, 2006), p. 10. 
Sordos-Mudos también fue construído por el Emperador Don Pedro II tres años después, en 1857, en la ciudad de Río de Janeiro. Este instituto también era muy parecido al Institut National des Jeunes Sourds, fundado en París, en 1770 (87 años antes). ${ }^{6}$

Si hablamos de Escuelas Normales, la gran pregunta es: ¿quiénes eran los profesores de estos institutos? ¿Dónde se graduaban, ya que no habían Escuelas Normales aún en la colonia Brasil?. Cuando investigamos estos dos puntos, vemos que la formación de estos maestros no se realizaba en institutos o sitios nacionales. Al principio, esta formación se llevaba a cabo en otros países, sobre todo en Francia, donde existián institutos para no videntes y sordos que citamos antes.

En el caso de José Alvares de Azevedo, por ejemplo, que estudió en el Instituto de París, cuando él regresa a Brasil, en 1852, ya no existía en el Instituto Imperial de no videntes, una de sus actividades que era justamente dar clases particulares a personas ciegas. Se sabe también, que los alumnos del Instituto de no videntes podían trabajar como "repetidores" ${ }^{7} \mathrm{Y}$ después de dos años en esta función, podían ser profesores del Instituto. En el informe de 1872, encontramos que $81 \%$ de los estudiantes se quedaron en la institución como profesores.

Para la historia de la Educación especializada en Brasil, la fundación del Instituto para no videntes $y$, después, para los sordos, fueron y son iniciativas muy importantes porque definen claramente un lugar de educación para las personas con discapacidad. Contar con una perspectiva educativa era considerablemente distinto de ser objeto de atención asistencial y religiosa. Los institutos no son asilos para personas consideradas como anormales, ni son hospicios. Esto es muy marcante, por ejemplo, en el discurso de inauguración del Instituto de no videntes, por el Dr. José Francisco Sigaud. El doctor, hablaba literalmente que se trataba de un espacio de educación.

En el caso del Instituto para sordos, se observa también que el Instituto de París fue una referencia. Pero, son muy visibles los movimentos de las personas sordas para crearlo. Situación no dada solamente en Brasil, también en muchos países, el proceso fue igualar. Cuando los sordos estudiaban en un instituto más reconocido y después cuando regresaban a sus países o viajaban a otros sitios donde había sordos, realizaban una articulación política para fundar instituciones de educación.

Otro punto que es muy interesante es que en 1883, en el I Congreso de Instrucción Pública de la colonia, uno de los temas discutidos fue el currículo para formación de maestros para trabajar con sordos y no videntes. ${ }^{8}$

\footnotetext{
6 Marcos José da Silveira Mazzota. Educação especial no Brasil: História e políticas públicas. (São Paulo: Cortez, 2005), p. 28.

7 Gilberta de Martino Jannuzzi. A educação do deficiente no Brasil: Dos primórdios ao início do século XXI. (Campinas (SP): Autores Associados, 2006), p. 12.

8 Îdem, p. 15.
} 
Hoy, el instituto para sordos se llama Instituto Nacional de Educación de Sordos y hay un Colegio de educación bilingüe para alumnos sordos. Durante todo el tiempo de la educación básica y, como formación universitaria, hay un Curso Bilingüe de Pedagogía, donde los pedagogos y pedagogas aprenden también la lengua de signos. Por otro lado, el Instituto para no videntes se llama Instituto Benjamin Constant y tiene cursos para formación de maestros que desean trabajar con alumnos no videntes. Es también un instituto de investigación y de producción de conocimiento.

Más allá de la especificidad de estos dos institutos, es necesario comprender las Escuelas Normales para personas con discapacidad en Brasil en el contexto de las Escuelas Normales regulares. Como se sabe, las Escuelas Normales fueron necesarias porque en ellas se introdujo el tema de la instrucción de la población de las capas populares.

Como nos enseña Saviani, ${ }^{9}$ en Brasil el tema de las Escuelas Normales fue importante después de la proclamación de la República (1889), cuando se hace necesario estructurar la instrucción popular. El autor destaca seis momentos que fueron marcantes en la definición de la formación de maestros en Brasil a partir de entonces, distribuidos en los siguientes períodos: 1827-1890, 1890-1932, 19321939, 1939-1971, 1971-1996, 1996-2006. Trataremos algunos elementos que fueron relevantes para nuestro tema en estos intervalos históricos, sin la intención de agotar, al mismo tiempo, todas las realizaciones implementadas durante este trayecto.

\section{República, educación especializada y formación de profesores}

Entre los años de 1827 y 1890, más allá de los institutos imperiales que ya abordamos, tenemos la ley de las escuelas de primeras letras - que obligaba a los profesores a instruirse en el método de enseñanza mutua (Método Lancaster), con sus propios recursos. En 1834, la instrucción primaria se hace responsable de las provincias y se adopta, en general el modelo que existía en los países europeos, de las escuelas primarias. La provincia de Río de Janeiro crea la primera Escuela Normal en Niteroi, en 1835, después tenemos, aún en el siglo XIX Escuelas Normales en: “Bahía, 1836; Mato Grosso, 1842; São Paulo, 1846; Piauí, 1864; Río Grande do Sul, 1869; Paraná y Sergipe, 1870; Espírito Santo y Río Grande do Norte, 1873; Paraíba, 1879; Río de Janeiro (DF) y Santa Catarina, 1880; Goiás, 1884; Ceará, 1885; Maranhão, 1890". ${ }^{10}$ Algunas escuelas abrían, funcionaban un tiempo y después cerraban sus puertas y luego, volvían a trabajar. Era, pues, muy inestable. A partir de 1890, hay un fortalecimiento del modelo de las Escuelas Normales.

\footnotetext{
9 Demerval Saviani, "Formação de professores: aspectos históricos e teóricos do problema no contexto brasileiro", Revista Brasileira de Educação. Vol. 14 No. 40 (2009): 143.

10 Ídem, p. 144.
} 
De 1890 a 1932, hay una consolidación y expansión de las Escuelas Normales, teniendo como referencia la Reforma de la Escuela Normal Paulista (de São Paulo), que era una escuela modelo.

En 1926, es fundada la segunda institución escolar para no videntes en Brasil, y este Instituto se encontraba en Belo Horizonte (capital de Minas Gerais). Mas allá de enseñar a alumnos no videntes, este Instituto también tuvo cursos de formación de maestros en años posteriores, en el ámbito de la educación secundaria técnica (educación secundaria).

En 1929, el gobierno de Minas Gerais, invita a la psicóloga y educadora rusa, Helena Antipoff, para actuar en la Escuela de Perfeccionamiento Pedagógico, donde se iniciaba la reforma a la o de la Escuela Nueva del departamento. Su llegada fue importante no solamente para este departamento sino para todo el país, pues, a partir de los años 1930, se dio inicio a una serie de trabajos educativos destinados a personas con discapacidad.

En el período de 1932-1939 surge la organización de los institutos de educación, partiendo de dos reformas: Reforma Anisio Teixeira, en el Distrito Federal (entonces Río de Janeiro, 1932) y reforma Fernando de Azevedo, en São Paulo, 1933.

Entre los años de 1939 y 1971, hay una implantación de los cursos de formación de pedagogos y licenciados, y una consolidación de los modelos de las Escuelas Normales. Después de 1946, las Escuelas Normales deberían formar en dos modalidades: La primera, con duración de cuatro años, capacitaría profesores para la escuela primaria y los cursos serían ofrecidos en Escuelas Normales regionales. El segundo tipo, con duración de tres años, formaría también profesores para la educación primaria y sería ofrecida en Escuelas Normales e institutos de educación. Estos últimos tendrían en adjunto jardín de infancia y escuela primaria. Habría también, cursos de especialización de profesores primarios para educación especializada, diseño, artes aplicadas y, formación de administradores, inspectores y orientadores educacionales.

En los años 1940, la formación de maestros para la educación de alumnos discapacitados, se hacía en su mayoría en cursos de educación secundaria técnica. En esta época, algunas Escuelas Normales fueron transformadas en Institutos de educación. En estos Institutos, había, generalmente, cursos de formación, especialización y administración, como es el caso de la Escuela Caetano de Campos, en São Paulo, que era una Escuela Normal y después se transformó en un Instituto.

Algunos Institutos de Educación a partir de los años 1950 ofrecían cursos de formación para profesores que deseaban enseñar a alumnos con discapacidad cognitiva, sordos, ciegos. Estos cursos estaban dentro de los Cursos Normales de formación de maestros y podrían llevarse a cabo en Escuelas Normales o Institutos de Educación. 
En los cursos de especialización en discapacidad cognitiva se estudiaba, generalmente: ortofrenopedia, Psicología del niño retardado, Biología, Biotipología ehigiene mental, antropología pedagógica y medidas educacionales, trabajos manuales, música, diseño, educación física. Para la enseñanza de sordos: anatomía, patología de la audición y fonación, psicología, pedagogía y metodología, artes, orientación vocacional y rehabilitación. Para la enseñanza de no videntes: anatomía, fisiología, patología de los ojos y prevención de la ceguera, orientación vocacional y rehabilitación, efectos psicológicos de la ceguera, braille, metodología especializada y artes. ${ }^{11}$

La Ley de Directrices y Bases de la Educacion Nacional, de 1961, anterior al gobierno del Régimen Militar (dictadura), tiene un capítulo para la educación de los discapacitados, que en ese momento eran llamados de "excepcionales". En este capítulo, con muchos artículos, dice, entre otros aspectos, que - Su educación se debe mirar en la integración a la escuela regular; - Debe haber un perfeccionamiento de la educación, con desarrollo de técnicas y servicios de atención especializada; - Formación para los maestros, inversiones en las investigaciones, realización de congresos y conferencias. ${ }^{12}$

Entre los 25 años que hacen parte del período de 1971 a 1996, conforme intervalo propuesto por Saviani ${ }^{13}$, tenemos la emergencia de importantes hechos y tendencias. De 1964 a 1985 es tiempo de dictadura en Brasil y un período en el cual se observa una priorización de la dimensión técnica del quehacer educativo. La crítica a la hegemonía de esta concepción empieza a tener una mayor expresividad en la segunda mitad de los años de $1970^{14}{ }^{15}$. En estos años, hay una sustitución de la Escuela Normal por habilitación específica para el Magisterio. Al final de este período, hubo un consenso según el cual, el pedagogo debería graduarse para actuar en la educación de la primera infancia y en la escuela primaria. A partir de 1972, los cursos de especialización y de formación en educación especializada ocurren en ámbito de la educación superior de corta duración (Deliberación CEE n. 15/71). Como educación superior, estas habilitaciones se daban, generalmente, en los cursos de Pedagogía. Muchas veces, había una parte común y otra más especializada, destinada a la formación de maestros en alguna discapacidad. En algunos momentos de estos cursos de formación de educadores, encontramos, muchas veces: introducción a la Educación especializada, Psicología de desarrollo, Psicología del aprendizaje, educación primaria y secundaria. En la parte específica, encontramos habilitaciones para actuar con discapacitados de la comunicación, físicos, mentales, de videncia. ${ }^{16}$

11 Marcos José da Silveira Mazzota. Trabalho docente e formação de professores em educação especial (SP: EPU, 1993), p. 64.

12 “Lei no. 4.024, de 20 de dezembro de 1961" (http://www.planalto.gov.br/ccivil_03/leis/14024.htm (2 de marzo, 2014).

13 Ídem, ibídem.

14 Vera Maria Candau, “A formação de educadores: uma perspectiva multidimensional”, Em Aberto. Vol.: 1 No. 8 (1982): 19.

15 Julio Emilio Diniz-Pereira, "A construção do campo da pesquisa sobre formação de professores", Revista da FAEEBA - Educação e Contemporaneidade. Vol: 22 No. 40 (2013): 147.

16 Marcos José da Silveira Mazzota. Trabalho docente e formação de professores em educação especial (SP: EPU, 1993), p. 70-72. 
Desde el punto de vista de Masini, ${ }^{17}$ este cambio de orientación legal alterando la formación en nivel secundaria para nivel superior de corta duración - valoró la formación de los educadores en el ámbito de la Educación Especial, haciendo posible que su habilitación sobrepase los aspectos técnicos para una mayor cualificación teórica y práctica. También, las licenciaturas de corta duración tuvieron fuertes críticas por parte de diversos educadores y teóricos del campo, dado el carácter de simplificación y de agilidad de la formación docente que este acortamiento podía evitar, y que abriría una desvaloración de la profesión docente y de la propia educación como un derecho.

\section{Democratización de Brasil y educación como derecho público subjetivo: ¿cuáles son los desafíos para la formación de profesores y para la educación escolar de personas con deficiencia?}

El proceso de democratización vivenciado en Brasil en la década de 1980, trajo preguntas centrales para las políticas públicas brasileras. La designación de los recursos públicos, la calidad y el alcance de los servicios ofrecidos a la población, son puestos en regla, preguntándose por el hecho de un número significativo de brasileros que no eran asistidos por el Estado en sus necesidades básicas.

En este escenario, una de las instituciones fuertemente interrogadas es la escuela pública, considerada como elitista, tanto por la existencia de un número insuficiente de establecimentos de enseñanza en relación a la población en edad escolar, como por los altos índices de evasión de repitencia que se presentaban, en su gran mayoría, en los alumnos provenientes de las clases populares.

La repercusión de estos asuntos en la sociedad puede ser observada a partir del final de la década de 1970. Sin embargo, adquiere mayor repercusión en los años 1980, por medio de los movimientos sociales que reivindicaban la educación, de las manifestaciones de los docentes en el interior de sus organizaciones profesionales, de la producción académica que se constituye a partir de estos temas y en iniciativas gubernamentales que tenían como meta producir cambios en esta realidad.

Con la apertura del país después del régimen militar, la nueva Constitución Federal de 1988 es promulgada. En los capítulos dirigidos al tema educacional se destacan: - el deber del Estado de ampliar la educación básica (educación infantil, educación primaria y secundaria); - la tarea de que se definan obligaciones específicas a los municipios, estados y a la Unión; - la perspectiva de que el derecho a la escuela pública es un derecho público subjetivo, por tanto, inalienable. ${ }^{18}$ En el artículo 208 de la Constitución, la atención educacional

\footnotetext{
17 Elcie Aparecida Fortes Salzano. Masini, "A educação do portador de deficiência visual: as perspectivas do vidente e do não vidente". Em Aberto. No. 60 (1993): 64.

18 José Silverio Baia Horta, "Direito à educação e obrigatoriedade escolar". Cadernos de Pesquisa. No. 104 (1998): 26-27.
} 
especializada es garantizada como deber del Estado, debiendo ser disponible en carácter preferencial en la red regular de enseñanza.

Más recientemente, en el período que comprende los años de 1996 a 2006, tenemos una nueva Ley de Directrices y Bases de la Educación Nacional (LDBN, 1996). ${ }^{19}$ Según esta Ley, es necesario organizar la formación de maestros en cursos de licenciatura de graduación plena, ofrecidos en universidades y en Institutos de educación superior. La formación de profesores podrá ocurrir, también, en universidades integradas, universidades aisladas y centros universitarios. Para la escuela primaria, había como opción el Curso Normal Superior, que era un magisterio ampliado, con estatus de formación en grado superior. Este curso no continuó y el Curso de Pedagogía asumió esta obligación.

La LDBN dedica el capítulo "V" a la Educación Especial, conceptuándola en su artículo 58 como "[...] modalidad de educación escolar, ofrecida preferencialmente en la red regular de enseñanza, para educandos portadores de necesidades especiales". La Ley determina la pertinencia de servicios de apoyo especializado en la escuela regular para atención a las peculiaridades del alumnado de Educación Especial y prescribe la atención educacional en clases, escuelas o servicios especializados, para los casos en los cuales no sea posible la integración en la enseñanza común. Un dato nuevo introducido en la Ley es la oferta de Educación Especial en la Educación Infantil.

Analizando la LDBN, Carvalho ${ }^{20}$ resalta la abertura de la Ley para los procesos de formación continuada y alerta para la urgencia de que temáticas relacionadas a la Educación Especial sean contemplados en estos procesos formativos. Pero, en relación a la formación de las licenciaturas, ¿qué es posible indicar después de la introducción de la LDB de 2006? La Ley anticipa la existencia de profesores especializados y profesores capacitados para la integración, sin embargo, existen fallas en los procedimentos que programaron esta formación: si sería por medio de cursos de especialización, oferta de asignaturas durante las graduaciones o por habilitaciones específicas, como ocurre en algunas instituciones brasileras, como en el Curso de Pedagogía de la Universidad Estatal Paulista Júlio de Mesquita Filho, y en el Curso de licenciatura en Educación Especial de la Universidad Federal de São Carlos.

Es importante considerar, también, que se dieron cambios significativos a partir de mediados de los años de 1990 en lo que respecta al trayecto de escolaridad de los estudiantes que presentan alguna discapacidad. A partir de esta década, pasa, paulatinamente, de un modelo referenciado en la integración escolar de las personas con deficiencia - que presuponga una escolaridad en ambientes educacionales especializados para este grupo, pudiendo ser seguida o no de una integración en las escuelas y clases comunes - para una perspectiva de educación inclusiva, a partir de la cual se prioriza una trayectoria escolar

19 "Lei no. 9.394, de 20 de dezembro de 1961" (http://www.planalto.gov.br/ccivil_03/leis/19394.htm (20 de marzo, 2014).

20 Rosita Edler Carvalho, A nova LDB e a educação especial (Rio de Janeiro: WVA, 1997), p.80. 
preferencial o exclusiva en las escuelas comunes. Además del cambio de una perspectiva integradora para una perspectiva inclusiva, se verifica que las declaraciones y acuerdos internacionales pasan a tener una expresividad mucho mayor en la definición de las políticas nacionales destinadas a los alumnos con deficiencia o que presentan alguna dificultad escolar más significativa. Podemos citar, en este sentido, la Declaración Mundial de Educación para Todos (1990) y de la Declaración de Salamanca (1994). ${ }^{21}$

El período que comprende los años de 1996 a 2006, es marcado de un modo general, por la necesidad de implementar una serie de definiciones previstas en la LDBN de 2006. En el caso de la educación de personas con deficiencia, muchos temas inquietan a los gestores y profesionales de la educación, pues existe la urgencia de un reordenamiento de los servicios educacionales especializados, de la definición del recorrido escolar de este público, de la institucionalización de mecanismos de formación de profesores más adecuados con la realidad que se establecía.

A partir de 2006, nuevas orientaciones son fundamentales para el curso de Pedagogía, ahora no podemos decir que los Institutos superiores son importantes como en la LDBN, de 1996. Según la legislación de 2006, tres núcleos son fundamentales a la Pedagogía: un núcleo de estudios básicos, un núcleo de profundización y diversificación de estudios y un núcleo de estudios integradores.

Para las licenciaturas que forman profesores para la educación básica, de una manera general, hay también nuevas definiciones después de 2006. Los currículos tienen que tener como mínimo 1.800 horas para los contenidos curriculares de naturaleza científico-cultural, 400 horas para práctica de componente curricular, y 400 horas más para prácticas curriculares supervisionadas y 200 para otras actividades culturales y científicas.

En el 2008, el gobierno brasilero lanza el documento Política Nacional de Educación Especial en la Perspectiva de la Educación Inclusiva. En este, los servicios de apoyo especializado pasan a ser considerados como obligatoriamente complementares o suplementares, y no más como sustitutivos a la educación común; la educación especializada deja de ser considerada una modalidad que pasa todos los niveles, etapas y modalidades de enseñanza; y se instituye como obligatoria la oferta de atención educacional especializada en la propia escuela $o$ en el centro especializado, entre otros aspectos. Tales medidas producen modificaciones importantes en el recorrido educacional de los estudiantes con deficiencia, y hacen que crezca una demanda por la realización de procesos formativos más solidificados en esta área, en los cursos de formación de profesores existentes en el país. ${ }^{22}$

21 Mônica María Farid Rahme, "Inclusão e internacionalização dos direitos à educação: as experiências brasileira, norte-americana e italiana”. Educação e Pesquisa. Vol: 39 No. 1 (2013): 97.

22 Brasil, "Política nacional de Educação Especial na perspectiva da Educação Inclusiva" (Brasília: MEC/SEESP, 2008) portal.mec.gov. 


\section{La temática de la "deficiencia" como desafío para las licenciaturas de la Universidad Federal de Ouro Preto (UFOP)}

Sistematizar saberes y prácticas en los cursos de licenciatura, que permitan una mejor acogida de alumnos que presentan particularidades físicas, motoras, cognitivas o psíquicas en la escuela común, demanda procesos formativos comprometidos con un proyecto de sociedad más igualitario, lo que requiere reformulaciones alrededor de la función social de la escuela, de los mecanismos de gestión, de la prática docente y del currículo escolar. En la realidad brasilera, se desea, también, una valoración de la carrera docente y la construcción de políticas más sistemáticas de formación inicial, que apoyen a los profesores en la vivencia de su experiencia docente.

Teniendo como referencia este desafío, destacaremos en este artículo datos relativos a la presencia de la temática "discapacidad" en los cursos de licenciatura existentes en la Universidad Federal de Ouro Preto (UFOP), en los campos situados en las ciudades de Ouro Preto y Mariana ${ }^{23}{ }^{24}$. Aun la Universidad dispone de un tercer campo construído en la ciudad de João Monlevade, los cursos allí ofrecidos no se relacionan con el campo de formación docente, pero sí con las áreas de Ingeniería y Sistemas de la Información. Los cursos que compusieron nuestra investigación fueron: Artes Escénicas, Ciencias Biológicas, Filosofía, Física, Historia, Letras, Matemáticas, Música, Pedagogía y Química.

Se trata de una investigación realizada entre los años 2011 y 2013, cuyo objetivo era verificar si estos cursos dialogaban en términos curriculares, con la producción de conocimientos académicos sobre aspectos educacionales con respecto a la deficiencia. Para ello, se consultaron índices, programas de disciplina y proyectos de los cursos de graduación citados, y que eran ofrecidos en la modalidad presencial. En la versión completa de la investigación, consideramos, para efectos de análisis, los datos de las disciplinas electivas, que no son, todavía, tratados en este artículo.

A partir del análisis de los datos recolectados, se comprobó que siete asignaturas obligatorias trataban directa o indirectamente temáticas relacionadas a la deficiencia en la Universidad Federal de Ouro Preto, en la ciudad de Mariana. Se tratan de las siguientes asignaturas y cursos: Política y Gestión (Letras y Pedagogía), Psicología de la Educación I y II (Letras, Historia, Pedagogía), Fundamentos de la Educación: Sociología (Pedagogía e Historia) y Necesidades Educacionales Especiales (Pedagogía), Introducción a la Lengua Brasilera de Señales - LIBRAS (Letras y Pedagogía). En relación al campo de Ouro Preto, se observó que la disciplina Psicología de la Educación II, se encontraba presente

br/seesp/arquivos/pdf/politica.pdf (18 decembro, 2012).

23 Margareth Diniz et al. "Levantamento de dados nos currículos dos cursos da UFOP sobre a diversidade, inclusão e não exclusão educacional” (Pró-Ativa/PROGRAD: DEEDU/ICHS/UFOP, 2011), s/p.

24 Mônica María Farid Rahme et al. "A questão da diversidade e da inclusão educacional no Ensino Superior: um estudo sobre os currículos dos cursos da UFOP” (PROBIC-CNPQ-PROPP: DEEDU/ICHS/UFOP, 2013), s/p. 
en los siguientes cursos de Licenciatura: Biología, Química, Artes Escénicas y Educación Física.

Con base en estos datos, se puede indicar que el tema de la deficiencia no se encuentra totalmente ausente de los cursos de licenciatura investigados, aunque su presencia sea aún incipiente y puntual. Esto indica la necesidad de expandir y profundizar la aproximación de este tema en los cursos existentes, así como de evaluar la situación real de las asignaturas que se encuentran introducidas en las propuestas curriculares. En este sentido, es importante destacar que, de las asignaturas y cursos citados, apenas el curso de Pedagogía cuenta con una asignatura destinada específicamente a esta temática (Necesidades Educacionales Especiales), y que la disciplina de Introducción a LIBRAS es obligatoria solamente en los cursos de Pedagogía y Letras.

Por lo tanto, si la legislación brasilera orienta, por un lado, a la implementación de los temas relacionados a la educación de personas con deficiencia en las instituciones de enseñanza, como apoyo suplementario o complementario a la escolaridad. Por otro lado, no exige objetivamente la inserción de asignaturas sobre el tema en los currículos de los cursos que forman profesores. Esto explica una contradicción presente en los procesos de formación inicial, que ya deberían operar con prácticas y conocimientos que faciliten una escolaridad más abierta y efectiva a los alumnos que ingresan a la Educación Básica y que presentan particularidades durante su trayectoria escolar.

\section{CONCLUSIÓN}

Buscamos tratar a lo largo de este texto temas que permitan al lector construir una visión general sobre la interfaz formación de profesores y educación de alumnos con deficiencia en Brasil. Nos referenciamos en la división de períodos propuesta por Saviani ${ }^{25}$ y en la introducción de elementos específicos del campo de la educación especializada. Este trayecto nos permitió discutir cómo los procesos de formación de profesores están vinculados a definiciones relativas a la política educacional del país, y cómo su implementación es desafiadora.

Al momento de finalizar, reafirmamos la importancia de que la discusión sobre la discapacidad y los principios efectivos de la práctica sean introducidos en los currículos de las licenciaturas, y que los procesos de formación articulen efectivamente la teoría y la práctica para que los estudiantes se queden menos desorientados y menos precavidos con la inclusion al aula regular. Estamos de acuerdo, así, con Gatti, ${ }^{26}$ al señalar que los procesos de formación docente,

25 Demerval Saviani, "Formação de professores: aspectos históricos e teóricos do problema no contexto brasileiro", Revista Brasileira de Educação. Vol: 14 No. 40 (2009): 143.

26 Bernadete Angelina Gatti, "Formação inicial de professores para a educação básica: pesquisas e políticas educacionais", Estudos em Avaliação Educacional. Vol. 25 No 57 (2014): 40. 
deben facilitar que los futuros profesores traten con los alumnos reales que se encuentran en las escuelas, y no con estudiantes ficticios. Según la autora: "Una vez que esos alumnos no son seres abstractos, y sí seres que comparten su constitución con ambientes sociales cada vez más complejos, el trabajo de los profesores demanda comprensión más real sobre estos, sobre la propia institución-escuela en una formación que les permita tratar con las condiciones concretas de aprendizaje en los ambientes de las salas de clase"

Tales confrontaciones son fundamentales para que los procesos de formación de profesores absorban en sus currículos los saberes y las experiencias que fueron y han sido construídos en las escuelas sobre la escolaridad de los alumnos que experimentan particularidades físicas, motoras, sensoriales, psíquicas y cognitivas, afirmandos en su sistematización y en su socialización en los cursos de licenciatura. Además de eso, es importante que los cursos valoren didácticas que operan no solamente con el aprendizaje del conocimiento formal de las áreas, pero sí con los sentimientos que surgen de la práctica docente, inclusive, en relación a los sujetos que hacen presente la diferencia, como ocurre, muchas veces, con los alumnos que tienen alguna discapacidad.

\section{REFERENCIAS}

Candau, Vera María. "A formação de educadores: uma perspectiva multidimensional". Em Aberto. Vol: 1 No. 8 (1982): 19-21.

Carvalho, Rosita E. A nova LDB e a educação especial. Río de Janeiro: WVA, 1997.

Diniz, Margareth, Vieira, Ubiratan G., Rahme, Mônica María F., Raimundi, Iasmin C.y Brandão, Ricardo de A. Levantamento de dados nos currículos dos cursos da UFOP sobre a diversidade, inclusão e não exclusão educacional. Pró-Ativa/ PROGRAD: DEEDU / ICHS/UFOP, 2011.

Diniz-Pereira, Júlio Emilio. “A construção do campo da pesquisa sobre formação de professores". Revista da FAEEBA - Educação e Contemporaneidade. Vol. 22 No. 40 (2013): 145-154.

Gatti, Bernadete Angelina. "Formação inicial de professores para a educação básica: pesquisas e políticas educacionais". Estudos em Avaliação Educacional. Vol. 25 No 57 (2014): 24-54.

Horta, José Silverio B. "Direito à educação e obrigatoriedade escolar". Cadernos de Pesquisa. No. 104 (1998): 5-34.

Jannuzzi, Gilberta de Martino. A educação do deficiente no Brasil: Dos primórdios ao início do século XXI. 2a. Ed. Campinas (SP): Autores Associados, 2006.

Lemos, Francisco M. y Ferreira, Paulo F. Instituto Benjamin Constant: uma história centenária. Rio de Janeiro: Instituto Benjamin Constant, 1995. 200.156.28.7/ Nucleus/media/common/Downloads_Historia_IBC.doc, (10/3/2014). 
Masini, Elcie Aparecida F. S. "A educação do portador de deficiência visual: as perspectivas do vidente e do não vidente". Em Aberto. No. 60 (1993): 60-76.

Mazzota, Marcos J. S. Trabalho docente e formação de professores em educação especial. SP: EPU, 1993.

Mazzota, Marcos J. S. Educação especial no Brasil: História e políticas públicas. 5 a . ed. São Paulo: Cortez, 2005.

Rahme, Mônica María F., Diniz, Margareth y Maia, Eliana do N. L. A questão da diversidade e da inclusão educacional no Ensino Superior: um estudo sobre os currículos dos cursos da UFOP. PROBIC-CNPQ-PROPP: DEEDU/ICHS/UFOP, 2013.

Rahme, Mônica María F. "Inclusão e internacionalização dos direitos à educação: as experiências brasileira, norte-americana e italiana". Educação e Pesquisa. Vol: 39 No. 1 (2013): 95-110.

Saviani, Demerval. "Formação de professores: aspectos históricos e teóricos do problema no contexto brasileiro". Revista Brasileira de Educação. Vol: 14 No. 40 (2009): 143155.

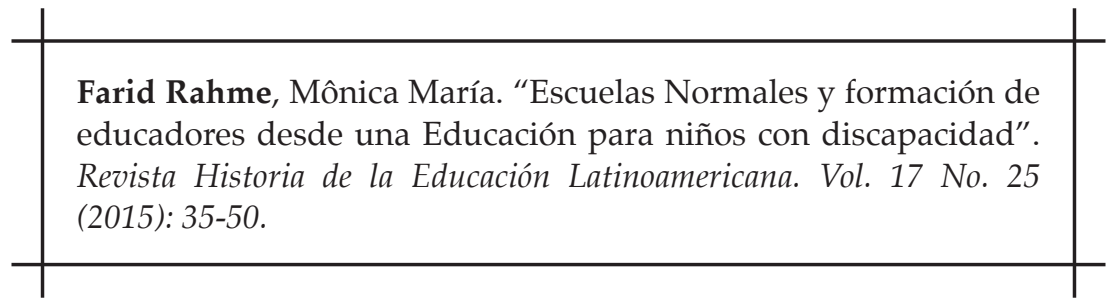




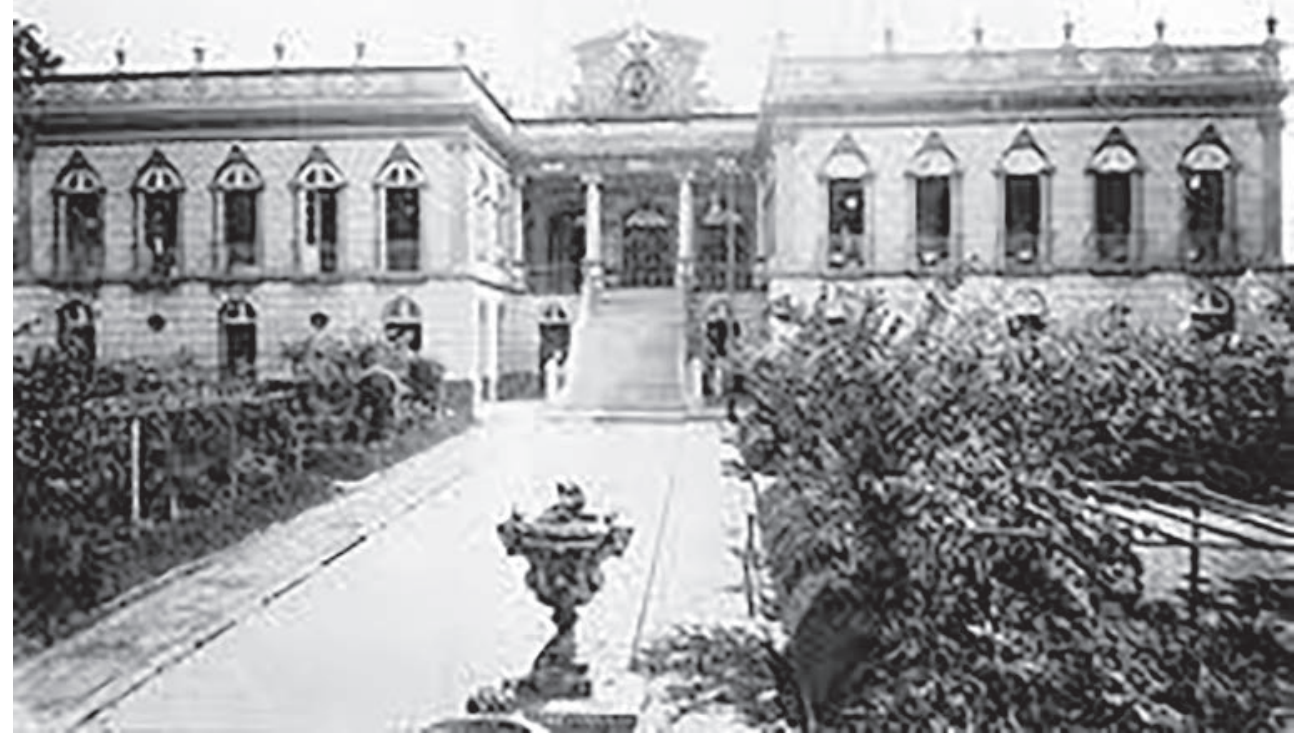

“Imperial Instituto de Meninos Cegos", construído por el Emperador Don Pedro II, en 1854, Río de Janeiro (Brasil).

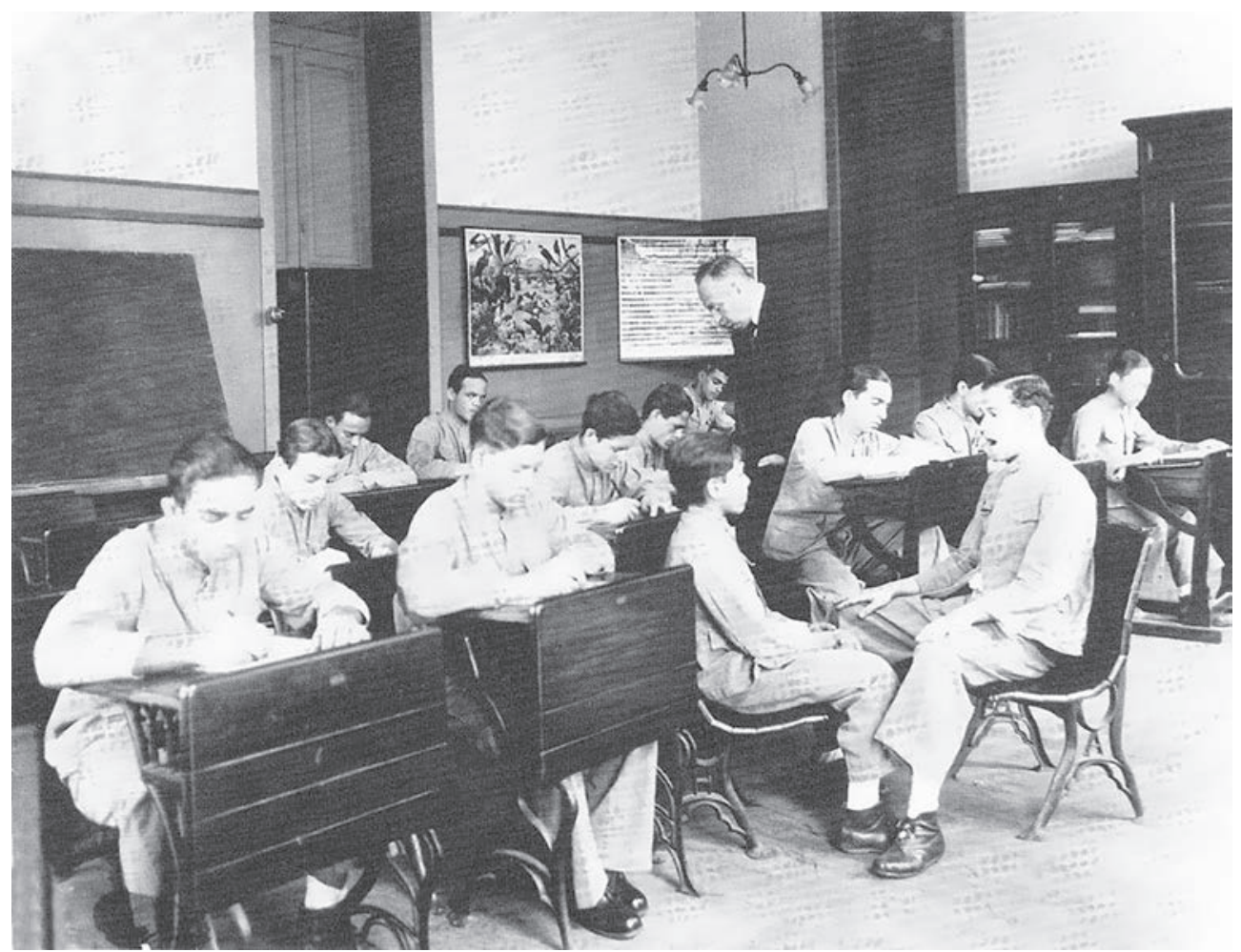

"Imperial Instituto de Sordos-Mudos", construído por el Emperador Don Pedro II, en 1857, Río de Janeiro (Brasil). 